\title{
Diagnosticando depressão em pacientes internados com doenças hematológicas: prevalência e sintomas associados
}

\author{
Diagnosing depression among hematological impatients: prevalence and \\ associated symptons \\ Letícia M. Furlanetto, Joanita Ângela Gonzaga Del Moral, Ana Heloísa B. Gonçalves, \\ Kenia Rodrigues e Maria Eduarda M. L. Polli Jacomino
}

\begin{abstract}
Resumo
Introdução: Não encontramos estudos avaliando o diagnóstico e a prevalência de depressão em pacientes hematológicos aqui no Brasil. Objetivo: Verificar a prevalência dos sintomas depressivos e quais deles mais se associam à depressão em pacientes internados com doenças hematológicas. Métodos: Num estudo transversal, 104 pacientes consecutivamente internados nos leitos da hematologia do Hospital Universitário da Universidade Federal de Santa Catarina (HU/UFSC) foram avaliados. Foram preenchidos questionários de variáveis sociodemográficas e de história psiquiátrica. O índice Charlson de co-morbidade (IC) foi usado para medir gravidade física. Foi aplicado, também, o inventário Beck de depressão (BDI). Aqueles que tiveram pontuação acima de 9 na soma dos 13 primeiros itens do BDI (BDI-13) foram considerados deprimidos. Também foi verificada a frequêencia caso fosse utilizada a escala completa com 21 itens (BDI-21), com ponto de corte 16/17. Resultados: As prevalências foram: $\mathrm{BDI}-13=25 \%$ e BDI-21 $=32,7 \%$. Após controle para fatores de confusão, os sintomas que permaneceram no modelo da regressão logística, indicando que melhor detectavam os deprimidos, foram sensação de fracasso, anedonia, culpa e fadiga. Conclusão: Cerca de um quarto a um terço dos pacientes internados com doenças hematológicas tinham sintomas depressivos significativos, e os sintomas que melhor os discriminaram foram sensação de fracasso, anedonia, culpa e fadiga.

Palavras-chave: sintomas depressivos, hematologia, pacientes internados, co-morbidade psiquiátrica, prevalência.
\end{abstract}

\begin{abstract}
Introduction: We did not find studies evaluating diagnosis and prevalence of depression in patients with hematological diseases in Brazil. Objective: To verify the prevalence of depressive symptoms and to identify which of them better help discriminate hematological inpatients who are depressed. Methods: A transversal study was conducted with 104 inpatients consecutively admitted to the hematology ward of Hospital Universitário of Universidade Federal de Santa Catarina. Data on sociodemographic variables and history of psychiatric disorders were collected. The Charlson Comorbidity Index $(\mathrm{Cl})$ was used to measure medical comorbidity. Depressive symptoms were assessed using the Beck Depression Inventory (BDI). Those who scored above 9 in the sum of the first 13 items (BDI-13) were considered depressed. We also evaluated prevalence using all the 21 items (BDI-21), by means of a cut-off score of 16/17. Results: Prevalences were: $\mathrm{BDI}-13=25 \%$ and $\mathrm{BDI}-21=32.7 \%$. After control for confounders, the symptoms that remained in the logistic regression model, indicating their ability to detect depressed patients, were sense of failure, anhedonia, guilt and fatigue. Conclusions: Around a quarter to a third of hematological inpatients had significant depressive symptoms and the ones that better discriminated depressed patients were sense of failure, anhedonia, guilt and fatigue.

Key words: depressive symptoms, hematology, medical inpatients, psychiatric comorbidity, prevalence.
\end{abstract}

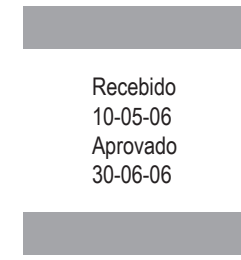

Centro de Ciências da Saúde da Universidade Federal de Santa Catarina (CCS/UFSC) (Furlanetto L, Del Moral JAG, Gonçalves AHB, Rodrigues K, Jacomino MEMLP)

0-05-06

Aprovado

Correspondência para: Letícia Maria Furlanetto

Departamento de Clínica Médica, $3^{\circ}$ andar, HU/UFSC - Caixa Postal 5199, Campus Universitário - 88040-970

- Florianópolis-SC - Tel./fax: (48) 3234-9756 - e-mail: leticiafurlanetto@yahoo.com.br 


\section{Introdução}

Evidências sugerem um aumento da freqüência de depressão nos pacientes com doenças físicas (de Jonge et al., 2006a). Além disso, indivíduos com sintomas depressivos desenvolvem mais comumente determinadas doenças: diabetes (Golden et al., 2004), hipertensão arterial (Everson et al., 2000) e acidente vascular encefálico (AVE) (Larson et al., 2001). Essa co-morbidade é preocupante porque um paciente clínico deprimido ajuda menos no tratamento (Lin et al., 2004), tem pior controle de sua doença de base (Lustman e Clouse, 2005), pior qualidade de vida (de Jonge et al., 2006b), maior prejuízo funcional (van Gool et al., 2005) e maior mortalidade (Colon et al., 1991; Furlanetto et al., 2000; Rumsfeld et al., 2005). A associação entre sintoma depressivo e doença clínica está bem estabelecida, sobretudo em cardiologia, endocrinologia e neurologia. Contudo existem poucos estudos sobre prevalência e diagnóstico da depressão em pacientes com doenças hematológicas, e não encontramos nenhum artigo com dados sobre a nossa realidade (realizado aqui no Brasil).

Existe ampla variação nas taxas de depressão em pacientes clínicos: de $3 \%$ a $75 \%$, dependendo do local de seleção (pacientes internados ou ambulatoriais), do modo de avaliação (definição de caso, instrumento, ponto de corte) ou das características específicas da doença física avaliada (Evans et al., 2005). Assim, caso seja usada uma entrevista estruturada e critérios diagnósticos para depressão maior em pacientes ambulatoriais clinicamente compensados, podemos encontrar taxas mais baixas. Por outro lado, se forem utilizadas escalas que medem sintomas depressivos em geral em pacientes internados, submetidos a diversos tratamentos, com dor e gravemente enfermos, essas taxas serão bem maiores. Outro fator que potencialmente pode levar a diferentes taxas consiste no fato de que sintomas vegetativos e somáticos causados unicamente pela doença de base (p. ex.: fadiga, insônia) podem ser confundidos com sintomas depressivos. Contudo um estudo recente mostrou a validade e a utilidade dos sintomas somáticos como critérios para depressão, mesmo nos pacientes com doenças físicas em geral (Simon e Von Korff, 2006). Neste trabalho, evidenciou-se correlação entre a gravidade dos sintomas somáticos (fadiga, alterações no apetite, peso, psicomotricidade e sono) e a gravidade da depressão. Além disso, houve melhora desses sintomas com o tratamento antidepressivo, tanto nos pacientes que tinham doenças físicas concomitantes como naqueles que não as possuíam. Nesse mesmo sentido uma pesquisa revelou que pacientes com linfoma de Hodgkin que estavam deprimidos apresentavam escores significativamente mais altos em um questionário de fadiga do que os não-deprimidos, independentemente de gravidade física (Loge et al., 2000).

São escassos os artigos sobre prevalência e sintomas associados à depressão em pacientes com doença hematológica. Além disso, alguns deles tiveram amostras pequenas e usaram diversos instrumentos ao mesmo tempo, sem esclarecer qual foi a definição de caso usada. Como exemplo citamos um estudo no qual foram avaliados 22 pacientes com leucemia, usando ao mesmo tempo a escala de Hamilton, o inventário Beck de depressão (BDI) e a escala de Zung. Esses autores não disseram como, mas relataram ter encontrado $18 \%$ de transtornos adaptativos depressivos. Referem também que tais transtornos foram mais freqüentes nos pacientes do sexo masculino (Hurtado et al., 1993).
Outros estudos com critérios um pouco mais claros, mas ainda com amostras pequenas, de 31 a 51 pacientes com leucemias e linfomas, revelaram taxas de depressão que variaram de $6 \%$ a 55\% (dos Santos et al., 1991; Hosaka et al., 1994; Montgomery et al., 2003). Assim, quando foi utilizada uma entrevista estruturada e os critérios da terceira revisão do Manual Diagnóstico e Estatístico de Transtornos Mentais (DSM-III-R), foi encontrada a taxa de $6 \%$ de pacientes com depressão maior e $23 \%$ com transtorno de ajustamento (Hosaka et al., 1994). Quando foi usada a escala de Zung com o ponto de corte de 39/40, deparou-se com a taxa de $55 \%$ de sintomas depressivos (Hosaka et al., 1994). Já em outra pesquisa, através da escala hospitalar de ansiedade e depressão (HAD), verificaram-se taxas de $51 \%$ ou $14 \%$, caso fossem utilizados os pontos de corte 13 ou 19, respectivamente (Montgomery et al., 2003). Nessa pesquisa houve associação entre idade mais avançada e depressão (Montgomery et al., 2003). Por fim, autores portugueses relataram que usaram uma entrevista semi-estruturada e os critérios do DSM-II-R, encontrando $30 \%$ de pacientes com "distúrbios adaptativos com humor depressivo ou misto (ansiedade e depressão)".

Já um estudo bem controlado e de critérios claros (DSMIV), realizado em 1.062 pacientes com neoplasias hematológicas internados para realização de transplante de medula óssea, revelou as taxas de $14 \%$ de depressão maior e $28 \%$ de transtorno de ajustamento (Prieto et al., 2002). Cerca de $30 \%$ de 290 pacientes internados com mieloma múltiplo assinalaram que "se sentiam muito deprimidos" no instrumento intitulado Multiple Affective Adjective Check List (Silberfarb et al., 1991). Uma pesquisa na qual 90 pacientes com linfoma de Hodgkin e não-Hodgkin foram questionados sobre sintomas depressivos, em média 32 meses após receberem o diagnóstico, revelou taxa de $20 \%$ de transtornos depressivos durante esse período (Devlen et al., 1987). Entretanto uma limitação do estudo é a possibilidade de ter ocorrido o viés das lembranças.

Em relação à depressão em doenças hematológicas nãoneoplásicas hão que se mencionar os estudos na doença falciforme. Uma pesquisa que avaliou 440 desses pacientes em nível ambulatorial com a escala de depressão do Centro para Estudos Epidemiológicos (CES-D) mostrou taxas de $43 \%$ e $18 \%$, com os pontos de corte de 16 e 27, respectivamente (Wison Schaeffer et al., 1999). Nesse grupo a depressão foi mais freqüente nas pacientes do sexo feminino e naqueles indivíduos que tinham mais baixa renda familiar. Outra investigação com 50 pacientes atendidos em um ambulatório para pacientes com doença falciforme revelou que $44 \%$ deles apresentavam sintomas depressivos, pelo menos leves, pelo BDI (Hasan et al., 2003).

Algumas pesquisas delineadas para avaliar a associação entre depressão e deficiência de vitamina $B_{12}$ e anemia mostraram resultados interessantes. Contudo não é possível, através de seus relatos, estabelecer a prevalência de depressão nesses grupos específicos, pois só referem a prevalência dessas deficiências em deprimidos e não-deprimidos (Penninx et al., 2000; Onder et al., 2005; Skarstein et al., 2005). Uma delas, na qual foram avaliadas 700 idosas na comunidade, revelou que a prevalência de deficiência de vitamina $B_{12}$ nas pacientes sem sintomas depressivos foi de $15 \%$, e naquelas com sintomas depressivos graves, 27\% (Penninx et al., 2000). Outra pesquisa, com 986 idosos, detectou anemia em $8 \%$ e $15 \%$ daqueles 
sem e com sintomas depressivos, respectivamente (Onder et al., 2005). Uma investigação realizada com 532 pacientes com neoplasias (não só hematológicas) também mostrou a relação entre níveis de hemoglobina e gravidade de sintomas depressivos (Skarstein et al., 2005). Há que se ressaltar, entretanto, que, apesar de esses trabalhos mostrarem a associação entre sintomas depressivos e deficiência de $B_{12}$ ou anemia, seus delineamentos não permitem que seja estabelecida a relação de causa e efeito. Podem significar que pacientes com anemia ou deficiência de $B_{12}$ apresentam mais sintomas depressivos; que pacientes deprimidos terminam por ter mais deficiência de $\mathrm{B}_{12}$ ou anemia; ou até mesmo que uma mesma causa possa levar aos dois desfechos (Onder et al., 2005). Outros autores não encontraram essa relação entre anemia e depressão no pós-parto (Paterson et al., 1994).

Como se pode notar, a literatura ainda é confusa e não temos pesquisas revelando dados da nossa realidade. Portanto os objetivos deste estudo são verificar a prevalência de sintomas depressivos e quais deles mais se associam à depressão em pacientes internados com doenças hematológicas.

\section{Métodos}

Trata-se de um estudo transversal no qual 104 pacientes consecutivamente internados nos leitos da hematologia do Hospital Universitário da Universidade Federal de Santa Catarina (HU/UFSC) foram avaliados dentro da primeira semana de internação. Foram colhidos dados sociodemográficos e feita a avaliação de história psiquiátrica pessoal e familiar, de acordo com o modelo realizado por Koenig et al. (Koenig et al., 1997). Foi aplicado o BDI (Beck et al., 1961), sendo utilizada a versão validada em português (Gorenstein e Andrade, 1996). Além disso, foi utilizado o índice de Charlson para medir a co-morbidade física dos pacientes (Charlson et al., 1987).

$\mathrm{Na}$ análise estatística, foram descritos os dados sociodemográficos, de história psiquiátrica e sintomas depressivos encontrados através de porcentagens, médias e \pm , conforme apropriado. Foram descritas também as freqüências de sintomas depressivos moderados a graves (escores 2 e 3 em cada item do BDI). Foram considerados deprimidos os indivíduos que tiveram pontuação acima de 10 na subescala cognitivo-afetiva, composta dos primeiros 13 itens do BDI (BDI-13). Essa subescala é preconizada por Beck em seu manual (Beck e Steer, 1993) para ser utilizada em pacientes com doenças físicas, tendo sido recentemente validada com essa finalidade em pacientes internados no Hospital Geral aqui no Brasil (Furlanetto et al., 2005). Somente a título de comparação, foi verificada também a prevalência caso fosse utilizada a escala completa de 21 itens (com ponto de corte 16/17). Também foram observadas as prevalências caso fossem avaliados separadamente pacientes com e sem neoplasias.

A seguir foram comparados os pacientes considerados deprimidos (de acordo com o BDI-13) com aqueles não-deprimidos em relação a variáveis sociodemográficas, história prévia de depressão, co-morbidade física e os itens do BDI. Para essa análise, as variáveis categóricas foram comparadas usando-se o teste do qui-quadrado, e as variáveis ordinais e contínuas foram avaliadas usando-se teste $t$ de Student para médias independentes ou o teste exato de Fischer, conforme apropriado. Foram considerados estatisticamente significativos os valores de $p \leq 0,05$. As variáveis que apresentaram diferença estatisticamente significativa foram utilizadas na análise multivariada. Para verificar quais sintomas mais se associavam ao diagnóstico de depressão de acordo com o BDI-13 foi realizado o teste da regressão logística, com o método stepwise likelihood ratio.

O estudo foi aprovado pelo Comitê de Ética em Pesquisas com Seres Humanos da UFSC. Todos os participantes assinaram termo de consentimento após esclarecimento. Aqueles indivíduos que apresentavam co-morbidade psiquiátrica e que desejavam ser acompanhados através de interconsulta psiquiátrica assim o foram.

\section{Resultados}

Foram elegíveis 123 pacientes, e, desses, 19 foram excluídos por impossibilidade física (9), prejuízo cognitivo (8) ou recusa (2). A amostra $(n=104)$ foi composta predominantemente de pacientes do sexo feminino (55,8\%), brancos $(81,7 \%)$, casados (55,8\%), com média de idade de 48,1 $\pm 19,8$ anos, escolaridade média de 6,1 $\pm 3,7$ anos e renda familiar de 3,2 $\pm 3,5$ salários mínimos. A Tabela 1 detalha as características sociodemográficas da amostra.

Quinze pacientes relataram história prévia de depressão $(14,4 \%)$. A média do índice de Charlson foi 1,4 $\pm 1,8$. Em relação ao diagnóstico hematológico, a porcentagem de pacientes com doenças oncológicas $(52,9 \%)$ foi semelhante à dos pacientes da hematologia geral (sem neoplasias) $(47,1 \%)$ (Tabela 2).

A prevalência de sintomas depressivos moderados a

Tabela 1. Características sodiodemográficas da amostra $(n=104)$

\begin{tabular}{lcc}
\hline Característica & $\boldsymbol{n}$ & $\%$ \\
\hline Sexo & & \\
\hline Masculino & 46 & 44,2 \\
Feminino & 58 & 55,8 \\
Idade & & \\
\hline 14-64 anos & 75 & 72,1 \\
$\geq 65$ anos & 29 & 27,9 \\
Raça & & \\
\hline Branca & 85 & 81,7 \\
Negra & 9 & 8,7 \\
Parda & 10 & 9,6 \\
Estado civil & & \\
\hline Solteiro(a) & 18 & 17,3 \\
Casado(a)/coabitando & 58 & 55,8 \\
Separado(a)/divorciado(a) & 12 & 11,5 \\
Viúvo(a) & 16 & 15,4 \\
Escolaridade & & \\
\hline 0 anos (analfabeto) & 5 & 4,8 \\
1-4 anos & 41 & 39,4 \\
$5-8$ anos & 28 & 26,9 \\
$>8$ anos & 30 & 28,8 \\
Renda familiar (salários mínimos) & & \\
\hline $1-3$ & 75 & 72,1 \\
$4-6$ & 20 & 19,2 \\
$7-10$ & 7 & 6,7 \\
$>10$ & 2 & 1,9 \\
\hline
\end{tabular}


graves de acordo com o BDI-13 (subescala cognitivo-afetiva) foi de $25 \%$ e, de acordo com a escala completa (BDI-21), 32,7\%. Não houve diferenças estatisticamente significativas na prevalência de sintomas depressivos quando foram comparados os pacientes que apresentavam doenças onco-hematológicas (BDI-13 = 25,5\% e BDI-21 $=34,5 \%)$ com aqueles da hematologia geral $(\mathrm{BDI}-13=$ $24,5 \%$ e BDI-21 $=30,6 \%$ ).

A Tabela 3 descreve as freqüências de sintomas moderados a graves de acordo com cada item do BDI na amostra. Os sintomas mais freqüentes foram perda da libido (38\%) e irritabilidade (36\%).

Tabela 2. Diagnósticos hematológicos da amostra ( $n=104)$

\begin{tabular}{lcc}
\hline Diagnósticos & $\boldsymbol{n}$ & \% \\
\hline Onco-hematologia & $\mathbf{5 5}$ & $\mathbf{5 2 , 9}$ \\
\hline Leucemias agudas & 24 & 23,1 \\
Linfomas & 19 & 18,2 \\
Síndrome mielodisplásica & 4 & 3,8 \\
Mieloma múltiplo & 3 & 2,9 \\
Policitemia vera & 2 & 1,9 \\
Leucemia linfocítica crônica & 1 & 1 \\
Mielofibrose & 1 & 1 \\
Leucemia de células dendríticas & 1 & 1 \\
Hematologia geral & 49 & $\mathbf{4 7 , 1}$ \\
\hline Anemias & 35 & 33,7 \\
Distúrbio de plaquetas & 9 & 8,7 \\
Hiperesplenismo & 3 & 2,9 \\
Distúrbio de leucócitos & 2 & 1,9 \\
\hline
\end{tabular}

Tabela 3. Freqüência de sintomas depressivos moderados a graves em pacientes hematológicos internados, de acordo com o Inventário Beck de Depressão $(n=104)$

\begin{tabular}{lcccc}
\hline Item/sintoma & \multicolumn{3}{c}{ Não } & \multicolumn{3}{c}{ Sim $^{*}$} \\
\hline 1. Tristeza & $\boldsymbol{n}$ & $\%$ & $\boldsymbol{n}$ & $\%$ \\
2. Desesperança & 86 & 83 & 18 & 17 \\
3. Sensação de fracasso & 94 & 90 & 10 & 10 \\
4. Anedonia & 87 & 84 & 17 & 16 \\
5. Culpa & 89 & 86 & 15 & 14 \\
6. Sentimento de punição & 90 & 87 & 14 & 13 \\
7. Desgosto consigo & 83 & 80 & 21 & 20 \\
8. Auto-acusação & 94 & 90 & 10 & 10 \\
9. Ideação suicida & 76 & 73 & 28 & 27 \\
10. Choro fácil & 98 & 94 & 6 & 6 \\
11. Irritabilidade & 88 & 85 & 16 & 15 \\
12. Perda do interesse nas & 67 & 64 & 37 & 36 \\
pessoas & 97 & 93 & 7 & 7 \\
13. Indecisão & 81 & 78 & 23 & 22 \\
14. Mudança de imagem do & 82 & 79 & 22 & 21 \\
corpo & 71 & 68 & 33 & 32 \\
15. Dificuldade no trabalho & 76 & 73 & 28 & 27 \\
16. Insônia & 71 & 68 & 33 & 32 \\
17. Fadiga & 85 & 82 & 19 & 18 \\
18. Falta de apetite & 71 & 68 & 33 & 32 \\
19. Perda de peso & 79 & 76 & 25 & 24 \\
20. Preocupação somática & 64 & 62 & 40 & 38 \\
21. Perda de libido &
\end{tabular}

${ }^{*}$ Sim corresponde aos sintomas que foram assinalados como moderados (2) ou graves (3) no inventário Beck de depressão.
$\mathrm{Na}$ análise bivariada, as variáveis que mostraram diferenças estatisticamente significativas entre deprimidos e não-deprimidos foram baixa escolaridade $(p=0,04)$, história de depressão $(p<0,001)$, índice Charlson de co-morbidade $(p=0,04)$ e todos os itens do BDI (todos com $p<0,01$ ). Portanto esses itens foram inseridos na análise multivariada. Após controle para fatores de confusão, os sintomas depressivos que permaneceram no modelo da regressão logística indicando que melhor ajudavam a diagnosticar depressão foram: sensação de fracasso, anedonia, culpa e fadiga (Tabela 4)

Tabela 4. Itens do inventário Beck de depressão que permaneceram no modelo da regressão logística*

\begin{tabular}{ll}
\hline Itens/sintomas & $\operatorname{Exp}(\mathbf{B})^{* *}$ \\
\hline 3. Sensação de fracasso & 1,24 \\
4. Anedonia & 4,12 \\
6. Culpa & 1,73 \\
17. Fadiga & 1,44 \\
\hline
\end{tabular}

*Foram inseridos na análise todos os itens do inventário Beck de depressão; **escolaridade + história prévia de depressão; * $\operatorname{Exp}(B)=$ exponencial de beta (corresponde ao aumento da chance de ter o diagnóstico de depressão com o aumento de cada ponto no sintoma em questão).

\section{Discussão}

Nosso estudo mostrou que $25 \%$ a $32,7 \%$ dos pacientes internados com doenças hematológicas apresentavam sintomas depressivos moderados a graves. Não houve diferença na freqüência de sintomas depressivos entre os pacientes hematológicos com e sem doenças oncológicas. Além disso, depois de controle para possíveis fatores de confusão (gravidade física, escolaridade e história de depressão), os sintomas que mais se associaram ao diagnóstico de depressão foram: sensação de fracasso, anedonia, culpa e fadiga.

Essa prevalência de sintomas depressivos em torno de $30 \%$ é semelhante à encontrada em vários estudos (dos Santos et al., 1991; Silberfarb et al., 1991; Wison Schaeffer et al., 1999). Contudo é mais alta do que as taxas de $6 \%$ e $14 \%$ evidenciadas em duas investigações que utilizaram entrevistas estruturadas e os critérios do DSM-IV para depressão maior (Hosaka et al., 1994; Prieto et al., 2002). Por outro lado, é mais baixa que as taxas de $44 \%, 51 \%$ e $55 \%$, encontradas quando foram utilizadas escalas (Hosaka et al., 1994; Hasan et al., 2003; Montgomery et al., 2003). Provavelmente as diferenças são decorrentes do modo de avaliação. É possível que muitos indivíduos deprimidos não cheguem a preencher critérios para depressão maior, mas já tenham sofrimento psíquico suficiente para obter pontuação nas escalas de depressão. Além disso, dependendo do ponto de corte utilizado na escala para detectar os casos, a prevalência pode sofrer variação. Esse provavelmente foi o motivo da diferença em relação ao único estudo que utilizou o BDI (mesmo instrumento que usamos) e encontrou $44 \%$ de sintomas depressivos. Esses autores utilizaram um ponto de corte mais baixo (9) com a escala completa (21 itens), incluindo, portanto, pacientes com síndromes depressivas leves (Hasan et al., 2003). 
Como significado clínico de nossos achados ressaltase que o conhecimento dos sintomas que mais se associaram à depressão pode ser útil para aumentar a detecção dessa co-morbidade e para melhor compreendermos os pacientes hematológicos deprimidos. A sensação de fracasso pode estar associada não só à depressão, mas também à desmoralização que esses pacientes sentem. Esse constructo elaborado por Frank se refere à perda da autoconfiança que pode ocorrer em pessoas com situação de vida desfavorável (Dohrenwend et al., 1980). A própria doença hematológica e seus tratamentos geram uma série de alterações no funcionamento e na aparência, deixando o indivíduo com a sensação de ser incapaz, um peso para todos. Isso provavelmente funciona como estressor importante que faz precipitar episódios depressivos naqueles que são vulneráveis à depressão. Uma vez iniciada, a depressão tem como um de seus sintomas a baixa auto-estima, o que agrava ainda mais a sensação de fracasso dos pacientes, e o círculo vicioso é mantido. A anedonia (perda de interesse e prazer) é um sintoma central da depressão e sabidamente responde a tratamentos farmacológicos. Embora pacientes clínicos estejam impossibilitados para muitas atividades prazerosas, aqueles que não estão deprimidos ainda conseguem imaginar ter prazer com o que antes gostavam (Furlanetto e Brasil, 2006). É interessante notar que já em 1917, em seu texto intitulado Luto e Melancolia, Freud referia que o sintoma culpa ajuda a diferenciar o luto (tristeza pela perda) da doença (melancolia) (Freud, 1953). Fadiga é um sintoma muito freqüente em pacientes com doenças hematológicas, sobretudo naqueles que estão anêmicos, com neoplasias e fazendo tratamentos como quimioterapia e radioterapia (Devlen et al., 1987; Sobrero et al., 2001). Contudo, quando a fadiga está associada a humor depressivo ou anedonia e excede o esperado para a gravidade da doença, deve levantar a suspeita de que esteja sendo piorada pela depressão (von Ammon Cavanaugh, 1995). A fadiga é sintoma que pode piorar a qualidade de vida e funcionar como um estressor a mais, precipitando ou agravando a depressão. Além disso, existem medidas, como a atividade física no leito, que podem ajudar a preveni-la (Dimeo et al., 1999).

\section{Referências}

Beck AT, Steer RA. Beck Depression Inventory Manual. San Antonio: Psychological Corporation; 1993.

Beck AT, Ward CH, Mendelson M, Mock J, Erbaugh J. An inventory for measuring depression. Arch Gen Psychiatry, 4: 561-71, 1961.

Charlson ME, Pompei P, Ales KL, MacKenzie CR. A new method of classifying prognostic comorbidity in longitudinal studies: development and validation. J Chron Dis, 40: 373-83, 1987.

Colon EA, Callies AL, Popkin MK, McGlave PB. Depressed mood and other variables related to bone marrow transplantation survival in acute leukemia. Psychosomatics, 32: 420-5, 1991.

de Jonge P, Kempen GI, Sanderman R, et al. Depressive symptoms in elderly patients after a somatic illness event: prevalence, persistence, and risk factors. Psychosomatics, 47: 33-42, 2006a.
Algumas limitações desse estudo merecem ser apontadas: 1) o uso de escalas permite verificar a presença de sintomas depressivos auto-relatados, mas não substitui 0 diagnóstico feito por profissional experiente utilizando entrevista estruturada e critérios diagnósticos estabelecidos. Entretanto, uma vez que a simples presença de sintomas depressivos já está associada a pior prognóstico (Furlanetto et al., 2000), o seu conhecimento pode ser útil para implementar medidas de prevenção e tratamento; 2) existe sobreposição dos sintomas depressivos àqueles causados pela doença hematológica, não sendo possível estabelecer a origem exata de cada um. A fadiga encontrada nesse grupo provavelmente teve várias causas concomitantes: aumento do metabolismo (febre, neoplasia, etc.); diminuição de substrato (anemia, hipoxemia, plaquetopenia ou baixo estado nutricional); liberação de substâncias que alteram 0 metabolismo ou o funcionamento dos músculos (citocinas, lactato) e depressão propriamente dita em alguns casos. Contudo, como já demonstrado, existe maior risco de fadiga em pacientes com doença de Hodgkin que estão deprimidos (Loge et al., 2000). Portanto, o raciocínio mais provável quando existe fadiga em excesso ao esperado é que ela seja oriunda da doença, dos tratamentos e da depressão concomitantemente.

\section{Conclusão}

Cerca de um quarto a um terço dos pacientes internados com doenças hematológicas apresentaram sintomas depressivos de intensidade moderada a grave. Eles foram mais freqüentes em indivíduos com história prévia de depressão, baixa escolaridade e maior co-morbidade física. Os sintomas que melhor ajudaram a detectar pacientes com síndromes depressivas moderadas a graves foram: sensação de fracasso, anedonia, culpa e fadiga.

São necessários estudos verificando se medidas visando a precocemente detectar e abordar esses sintomas podem contribuir para melhorar a qualidade de vida e o prognóstico desses pacientes. de Jonge P, Spijkerman TA, van den Brink RH, Ormel J. Depression after myocardial infarction is a risk factor for declining health-related quality of life and increased disability and cardiac complaints at 12 months. Heart, 92: 32-9, 2006b.

Devlen J, Maguire P, Phillips P, Crowther D, Chambers H. Psychological problems associated with diagnosis and treatment of lymphomas. I: Retrospective study. Br Med J (Clin Res Ed), 295: 953-4, 1987.

Dimeo FC, Stieglitz RD, Novelli-Fischer U, Fetscher S, Keul J. Effects of physical activity on the fatigue and psychologic status of cancer patients during chemotherapy. Cancer, 85: 2273-7, 1999.

Dohrenwend BP, Shrout PE, Egri G, Mendelsohn FS. Nonspecific psychological distress and other dimensions of psychopathology. Measures for use in the general population. Arch Gen Psychiatry, 37: 1229-36, 1980. 
dos Santos MJ, Pimentel P, Monteiro JM, et al. Psychiatric disorders in hospitalized patients with hematologic neoplasms. Acta Med Port, 4: 5-8, 1991.

Evans DL, Charney DS, Lewis L, et al. Mood disorders in the medically ill: scientific review and recommendations. Biol Psychiatry, 58: 175-89, 2005

Everson SA, Kaplan GA, Goldberg DE, Salonen JT. Hypertension incidence is predicted by high levels of hopelessness in Finnish men. Hypertension, 35: 561-7, 2000.

Freud S. Luto e melancolia. In: Obras Completas de Sigmund Freud. Rio de Janeiro: Editora Delta; 1953.

Furlanetto LM, Brasil MAA. Diagnosticando e tratando a depressão no paciente com doença clínica. J Bras Psiq, 55: 8-19, 2006.

Furlanetto LM, Mendlowicz MV, Bueno JR. The validity of the Beck Depression Inventory-Short Form as a screening and diagnostic instrument for moderate and severe depression in medical inpatients. J Affect Disord, 86: 87-91, 2005.

Furlanetto LM, von Ammon Cavanaugh S, Bueno JR, Creech SD, Powell $\mathrm{LH}$. Association between depressive symptoms and mortality in medical inpatients. Psychosomatics, 41: 426-32, 2000.

Golden SH, Williams JE, Ford DE, et al. Depressive symptoms and the risk of type 2 diabetes: the Atherosclerosis Risk in Communities study. Diabetes Care, 27: 429-35, 2004.

Gorenstein C, Andrade L. Validation of a Portuguese version of the Beck Depression Inventory and the State-Trait Anxiety Inventory in Brazilian subjects. Braz J Med Biol Res, 29: 453-7, 1996.

Hasan SP, Hashmi S, Alhassen M, Lawson W, Castro O. Depression in sickle cell disease. J Natl Med Assoc, 95: 533-7, 2003.

Hosaka T, Aoki T, Ichikawa Y. Emotional states of patients with hematological malignancies: preliminary study. Jpn J Clin Oncol, 24: 186-90, 1994.

Hurtado F, Martin G, Sanz MA. Leukemia and mental health: psychological disturbances, predisposing actors, precipitating conditions and psychotherapeutic approach. Sangre (Barc), 38: 429-34, 1993.

Koenig HG, George LK, Meador KG. Use of antidepressants by nonpsychiatrists in the treatment of medically ill hospitalized depressed elderly patients. Am J Psychiatry, 154: 1369-75, 1997.

Larson SL, Owens PL, Ford D, Eaton W. Depressive disorder, dysthymia, and risk of stroke: thirteen-year follow-up from the Baltimore epidemiologic catchment area study. Stroke, 32: 1979-83, 2001.

Lin EH, Katon W, Von Korff M, et al. Relationship of depression and diabetes self-care, medication adherence, and preventive care. Diabetes Care, 27: 2154-60, 2004.
Loge JH, Abrahamsen AF, Ekeberg, Kaasa S. Fatigue and psychiatric morbidity among Hodgkin's disease survivors. J Pain Symptom Manage, 19: 91-9, 2000.

Lustman PJ, Clouse RE. Depression in diabetic patients: the relationship between mood and glycemic control. J Diabetes Complications, 19: 113-22, 2005.

Montgomery C, Pocock M, Titley K, Lloyd K. Predicting psychological distress in patients with leukaemia and lymphoma. J Psychosom Res, 54: 289-92, 2003.

Onder G, Penninx BW, Cesari M, et al. Anemia is associated with depression in older adults: results from the InCHIANTI study. J Gerontol A Biol Sci Med Sci, 60: 1168-72, 2005.

Paterson JA, Davis J, Gregory M, et al. A study on the effects of low haemoglobin on postnatal women. Midwifery, 10: 77-86, 1994.

Penninx BW, Guralnik JM, Ferrucci L, Fried LP, Allen RH, Stabler SP. Vitamin $B(12)$ deficiency and depression in physically disabled older women: epidemiologic evidence from the Women's Health and Aging Study. Am J Psychiatry, 157: 715-21, 2000.

Prieto JM, Blanch J, Atala J, et al. Psychiatric morbidity and impact on hospital length of stay among hematologic cancer patients receiving stem-cell transplantation. J Clin Oncol, 20: 1907-17, 2002.

Rumsfeld JS, Jones PG, Whooley MA, et al. Depression predicts mortality and hospitalization in patients with myocardial infarction complicated by heart failure. Am Heart J, 150: 961-7, 2005.

Silberfarb PM, Anderson KM, Rundle AC, Holland JC, Cooper MR, McIntyre OR. Mood and clinical status in patients with multiple myeloma. J Clin Oncol, 9: 2219-24, 1991.

Simon GE, Von Korff M. Medical co-morbidity and validity of DSM-IV depression criteria. Psychol Med, 36: 27-36, 2006.

Skarstein J, Bjelland I, Dahl AA, Laading J, Fossa SD. Is there an association between haemoglobin, depression, and anxiety in cancer patients? J Psychosom Res, 58: 477-83, 2005.

Sobrero A, Puglisi F, Guglielmi A, et al. Fatigue: a main component of anemia symptomatology. Semin Oncol, 28: 15-8, 2001.

van Gool CH, Kempen GI, Penninx BW, Deeg DJ, Beekman AT, van Eijk JT. Impact of depression on disablement in late middle aged and older persons: results from the Longitudinal Aging Study Amsterdam. Soc Sci Med, 60: 25-36, 2005.

von Ammon Cavanaugh S. Depression in the medically ill. Critical issues in diagnostic assessment. Psychosomatics, 36: 48-59, 1995.

Wison Schaeffer JJ, Gil KM, Burchinal M, et al. Depression, disease severity, and sickle cell disease. J Behav Med, 22: 115-26, 1999. 